\title{
Cognitive Neurodynamics in China
}

\author{
Rubin Wang • Fanji Gu
}

Published online: 22 July 2010

(C) Springer Science+Business Media B.V. 2010

Noticeable progress has been made in the field of Cognitive Neurodynamics in China these years thanks to the diligent work of Chinese scientists, which is a brand new horizon for them to explore and devote unceasingly. To promote the communication and cooperation between scientists of China and all over the world, two international conferences on the topic were organized and held in China successfully in the past 3 years. In order to deepen the academic exchanges, spark the valuable ideas and accelerate the cooperation process, we organize this special issue of Cognitive Neurodynamics, and look forward to your constant concern for the potential realm of science as well as contact with proper author to discuss the subject of interest to you.

R. Wang

East China University of Science and Technology,

Shanghai, China

F. Gu ( ()

Fudan University, Shanghai, China

e-mail: sicpb2004@fudan.edu.cn 